\title{
ON SUMS OF HANKEL OPERATORS ${ }^{1}$
}

\author{
PAUL A. FUHRMANN
}

ABSTRACT. Necessary and sufficient conditions are derived for the sum of two Hankel operators of closed range to have closed range. As a corollary we determine when two left invariant subspaces of $H^{2}$ have positive angle.

In this note we investigate the range of the sum of two Hankel operators. Let us denote by $\mathbf{T}$ the unit circle $\{\lambda:|\lambda|=1\}$ and by $D$ the open unit disc $\{\lambda:|\lambda|<1\} . H^{2}$ will denote the usual scalar Hardy space [5] identified also as the subspace of $L^{2}(\mathrm{~T})$ of functions having vanishing negative Fourier coefficients. We denote by $P_{H^{2}}$ the orthogonal projection of $L^{2}(\mathbf{T})$ onto $H^{2}$. Let $J$ be the unitary map in $L^{2}(\mathbf{T})$ defined by $(J f)\left(e^{i t}\right)=f\left(e^{-i t}\right)$. Let $\phi \in H^{\infty}$; the Hankel operator $H_{\phi}$ corresponding to $\phi$ is the bounded operator in $H^{2}$ defined by

$$
H_{\phi} f=P_{H^{2}} \phi J f \text { for all } f \in H^{2} \text {. }
$$

It is clear from the definition that (Range $\left.H_{\phi}\right)^{-}$is a left invariant subspace of $H^{2}$. In [3] the following theorem of D. N. Clark has been proved.

Theorem A. The Hankel operator $H$ has closed range if and only if $\phi$ has a representation $\phi=q g$ with $q$ an inner function and $g \in \bar{H}_{0}^{\infty}$, i.e. $g$ is bounded conjugate analytic vanishing at $\infty$, and such that there exists $a$ $\delta>0$ for which

$$
|G(z)|+|\tilde{q}(z)| \geq \delta \quad \text { for all } z \in D
$$

where $\tilde{q}(z)=(q(\bar{z}))^{-}$and $G\left(e^{i t}\right)=e^{-i t} g\left(e^{-i t}\right)$.

Received by the editors July 25, 1973 and, in revi sed form, October 1, 1973. AMS (MOS) subject classifications (1970). Primary 47B99; Secondary 47A15.

Key words and phrases. Hankel operators, invariant subspaces, inner functions, closed range.

1 This work was supported by the U. S. Office of Naval Research under the Joint Services Electronics Program by Contract N00014-67- A-0298-0006. 
It follows in this case that Range $H_{\phi}=\left\{q H^{2}\right\}^{\perp}$.

In particular if $H_{\phi}$ has closed range then $\phi$ is noncyclic for the backward (left) shift [1].

Let us consider now two functions $\phi_{i} \in H^{\infty}$ which are noncyclic for the backward shift. Then (Range $\left.H_{\phi_{i}}\right)^{-}$are proper left invariant subspaces and hence there exist inner functions $q_{i}$ for which $\left\{\text { Range } H_{\phi_{i}}\right\}^{\perp}=q_{i} H^{2}$.

Theorem B. (a) (Range $\left.H_{\phi_{1}+\phi_{2}}\right)^{-}=\left(\text {Range }\left(H_{\phi_{1}}+H_{\phi_{2}}\right)\right)^{-}=\left\{q_{1} q_{2} H^{2}\right\}^{\perp}$ if and only if $q_{1}, q_{2}$ have no common nontrivial inner factor.

(b) Suppose Range $H_{\phi_{i}}=\left\{q_{i} H^{2}\right\}^{\perp}$, then Range $H_{\phi_{1}+\phi_{2}}=\left\{q_{1} q_{2} H^{2}\right\}^{\perp}$ if and only if for some $\delta>0$ and all $z \in D$ we have $\left|q_{1}(z)\right|+\left|q_{2}(z)\right| \geq \delta$.

Proof. (a) (Range $\left.H_{\phi}\right)^{-}=\left\{q H^{2}\right\}^{\perp}$ implies, since $\phi \in\left\{q H^{2}\right\}^{\perp}$, that $\phi=$ $q g$ with $g \in \bar{H}_{0}^{\infty}$. Let $\tau_{q}: L^{2}(\mathrm{~T}) \rightarrow L^{2}(\mathrm{~T})$ be the unitary map given by

$$
\left(\tau_{q} f\right)\left(e^{i t}\right)=e^{-i t} \tilde{q}\left(e^{i t}\right) f\left(e^{-i t}\right)
$$

then $x\left(\left\{q H^{2}\right\}^{\perp}\right)=\left\{\tilde{q} H^{2}\right\}^{\perp}$ [2]. In particular (Range $\left.H_{\phi}\right)^{-}=\left\{q H^{2}\right\}^{\perp}$ if and only if $\tau \phi$ is a cyclic vector for the restricted right shift in $\left\{\tilde{q} H^{2}\right\}^{\perp}$ and this occurs if and only if $\tau \phi, \tilde{q}$ have no common nontrivial inner factor [4]. But

$$
(\tau \phi)\left(e^{i t}\right)=e^{-i t} q\left(e^{i t}\right) q\left(e^{-i t}\right) g\left(e^{-i t}\right)=G\left(e^{i t}\right) .
$$

So (Range $\left.H_{\phi}\right)^{-}=\left\{q H^{2}\right\}^{\perp}$ is equivalent to $\phi=q g$ with $G, \tilde{q}$ having no common nontrivial inner factor.

Assume now $q_{1}, q_{2}$ to have no common nontrivial inner factor. Then $\phi_{1}+\phi_{2}=q_{1} g_{1}+q_{2} g_{2}$, and on applying $\tau_{q_{1} q_{2}}$ we get

$$
G=\tau_{q_{1} q_{2}}\left(\phi_{1}+\phi_{2}\right)=\tilde{q}_{2} G_{1}+\tilde{q}_{1} G_{2}
$$

and

$$
\text { (Range } \left.\left(H_{\phi_{1}}+H_{\phi_{2}}\right)\right)^{-}=\left(\text {Range } H_{\phi_{1}+\phi_{2}}\right)^{-}=\left\{q_{1} q_{2} H^{2}\right\}^{\perp}
$$

if and only if $G, \widetilde{q}_{1} \widetilde{q}_{2}$ have no common nontrivial inner factor. Suppose they have a common nontrivial inner factor $\psi$. Then $\psi \mid G$ and $\psi \mid \tilde{q}_{1} \tilde{q}_{2}$. Without loss of generality we may assume $\psi \mid \tilde{g}_{1}$. But then $\psi \mid \tilde{q}_{2} G_{1}$ and since $\psi$ and $G_{1}$ have no common inner factor by the assumption (Range $\left.H_{\phi}\right)^{-}=$ $\left\{q_{1} H^{2}\right\}^{\perp}$, then $\psi \mid \tilde{q}_{2}$ contrary to the assumption that $q_{1}, q_{2}$ have no common 
inner factor. Thus (Range $\left.H_{\phi_{1}+\phi_{2}}\right)^{-}=\left\{q_{1} q_{2} H^{2}\right\}^{\perp}$.

Conversely assume (Range $\left.H_{\phi_{1}+\phi_{2}}\right)^{-}=\left\{q_{1} q_{2} H^{2}\right\}^{\perp}$; then $\phi_{1}+\phi_{2}=$ $q_{1} q_{2} g$ for some $g \in \bar{H}_{0}^{\infty}$ and $G, \tilde{q}_{1} \tilde{q}_{2}$ have no common inner factor. Suppose $q_{1}, q_{2}$ have a common inner factor $\psi$. Then $\tilde{\psi} \mid \tilde{q}_{i}$. Hence $\tilde{\psi} \mid\left(\tilde{q}_{2} G_{1}+\tilde{q}_{1} G_{2}\right)$ that is $\tilde{\psi} \mid \tilde{G}$. But this contradicts the assumption that (Range $\left.H_{\phi_{1}+\phi_{2}}\right)^{-}=$ $\left\{q_{1} q_{2} H^{2}\right\}^{\perp}$.

(b) Let us assume not that range $H_{\phi}=\left\{q_{i} H^{2}\right\}^{\perp}, i=1,2$; then there exists a $\delta>0$ such that $\left|G_{i}(z)\right|+\left|q_{i}(z)\right| \geq \delta$ for all $z \in D$. Assume that for some $\delta_{1}>0$ we have $\left|q_{1}(z)\right|+\left|q_{2}(z)\right| \geq \delta_{1}$ for all $z \in D$. We will show that Range $H_{\phi_{1}+\phi_{2}}=\left\{q_{1} q_{2} H^{2}\right\}^{\perp}$. For this it suffices that $|G(z)|+$ $\left|\tilde{q}_{1} \tilde{q}_{2}(z)\right| \geq \delta_{2}>0$ for all $z$ in $D$. If this condition is not satisfied there exists a sequence $\left\{z_{n}\right\}$ in $D$ for which $G\left(z_{n}\right) \rightarrow 0$ and $\tilde{q}_{1}\left(z_{n}\right) \tilde{q}_{2}\left(z_{n}\right) \rightarrow 0$. By passing to a subsequence we may assume without loss of generality that $\tilde{q}_{1}\left(z_{n}\right) \rightarrow 0$. Since $G(z)=\tilde{q}_{1} G_{2}+\tilde{q}_{2} G_{1}$, it follows that $\tilde{q}_{2}\left(z_{n}\right) G_{1}\left(z_{n}\right) \rightarrow 0$. Now $G_{1}\left(z_{n}\right) \rightarrow 0$ is ruled out by $\left|G_{1}(z)\right|+\left|\tilde{q}_{1}(z)\right| \geq \delta$ whereas $\tilde{q}_{2}\left(z_{n}\right) \rightarrow 0$ is ruled out by $\left|q_{1}(z)\right|+\left|q_{2}(z)\right| \geq \delta_{1}$. So indeed Range $H_{\phi_{1}+\phi_{2}}=\left\{q_{1} q_{2} H^{2}\right\}^{\perp}$.

Conversely assume Range $H_{\phi_{1}+\phi_{2}}=\left\{q_{1} q_{2} H^{2}\right\}^{\perp}$. Then $G=\tilde{q}_{1} G_{2}+$ $\tilde{q}_{2} G_{1}$ and $|G(z)|+\left|\tilde{q}_{1} \tilde{q}_{2}(z)\right| \geq \delta$; i.e.

$$
\left|\tilde{q}_{1}(z) G_{2}(z)+\tilde{q}_{2}(z) G_{1}(z)\right|+\left|\tilde{q}_{1} \tilde{q}_{2}(z)\right| \geq \delta>0
$$

for all $z$ in $D$. But since $G_{i} \in H^{\infty}$, this implies $\left|q_{1}(z)\right|+\left|q_{2}(z)\right| \geq \delta_{1}>0$ for all $z$ in $D$.

Given any proper left invariant subspace $K$ of $H^{2}$ then $K$ is the range of a Hankel operator in $H^{2}$. In fact by Beurling's theorem, $K=\left\{q H^{2}\right\}^{\perp}$ for some inner function $q$. Let $\phi(z)=(q(z)-q(0)) / z$; then $\phi \in H^{\infty} \cap K$ and it is simple to check that, by Theorem $A$, Range $H_{\phi}=\left\{q H^{2}\right\}^{\perp}$. It is trivial that $\left\{q_{1} H^{2}\right\}^{\perp} \cap\left\{q_{2} H^{2}\right\}^{\perp}=\{0\}$ if and only if $q_{1}, q_{2}$ have no common nontrivial inner factor.

Corollary 1. Let $q_{1}, q_{2}$ be inner functions; then $\left\{q_{1} H^{2}\right\}^{\perp}+\left\{q_{2} H^{2}\right\}^{\perp}=$ $\left\{q_{1} q_{2} H^{2}\right\}^{\perp}$ if and only if there exists a $\delta>0 \cdot$ such that $\left|q_{1}(z)\right|+\left|q_{2}(z)\right| \geq \delta$ for all $z \in D$.

Now it is well known $\left[6\right.$, p. 243] that the sum of two subspaces $M_{1}, M_{2}$ of any Banach space, which satisfy $M_{1} \cap M_{2}=\{0\}$, is a closed subspace if and only if for some $d>0$, inf $\left\{\left\|x_{1}-x_{2}\right\|: x_{i} \in M_{i},\left\|x_{i}\right\|=1\right\} \geq d$. In a Hilbert space this condition is equivalent to $\sup \left\{\left|\left(x_{1}, x_{2}\right)\right|: x_{i} \in M_{i},\left\|x_{i}\right\|=1\right\}<1$ 
which can be interpreted geometrically as $M_{1}, M_{2}$ having a positive angle. Thus we get the following corollary.

Corollary 2. The angle between $\left\{q_{1} H^{2}\right\}^{\perp}$ and $\left\{q_{2} H^{2}\right\}^{\perp}$ is positive if and only if for some $\delta>0,\left|q_{1}(z)\right|+\left|q_{2}(z)\right| \geq \delta$ for all $z \in D$.

\section{REFERENCES}

1. R. G. Douglas, H. S. Shapiro and A. L. Shields, Cyclic vectors and invariant subspaces for the backward shift operator, Ann. Inst. Fourier (Grenoble) 20 (1970), fasc. 1, 37-76. MR 42 \#5088.

2. P. A. Fuhrmann, On the corona theorem and its application to spectral problems in Hilbert space, Trans. Amer. Math. Soc. 132 (1968), 55-66. MR 36 \#5751.

3. - Exact controllability and observability and realization theory in Hilbert space, J. Math. Anal. Appl. (to appear).

4. - On realization of linear systems and application to some questions of stability, Math. Systems Theory (to appear).

5. K. Hoffman, Banach spaces of analytic functions, Prentice-Hall Series in Modern Analysis, Prentice-Hall, Englewood Cliffs, N. J., 1962. MR 24 \#A2844.

6. A. E. Taylor, Introduction to functional analysis, Wiley, New York; Chapman \& Hall, London, 1958. MR 20 \#5411.

DIVISION OF ENGINEERING AND APPLIED PHYSICS, HARVARD UNIVERSITY, CAMBRIDGE, MASSACHUSETTS 02138

Current address: Department of Mathematics, University of the Negev, BeerSheva, Israel 